\title{
Safe Thrombolysis During Pregnancy for Recurrent Acute Ischaemic Stroke Due to Concomitant Isolated Left Ventricular Non-Compaction and Bilateral Foetal Posterior Communicating Arteries
}

Fatimah Osman Juma (DD

Leonard Mzee Ngunga

Wangari Waweru-Siika

Dilraj Singh Sokhi

Department of Medicine, Faculty of Health Sciences, Aga Khan University Medical College of East Africa, Nairobi, Kenya
Correspondence: Dilraj Singh Sokhi Aga Khan University Medical College of East Africa, Nairobi Campus, Room 405, 4th Floor East Tower Block, Third Avenue Parklands, P.O. Box 30270, Nairobi, 00100, GPO, Kenya

Tel +254 710559541

Email Dilraj.Sokhi@aku.edu

\begin{abstract}
Isolated left ventricular non-compaction (ILVNC) is a rare congenital cardiomyopathy and is associated with arrhythmias, heart failure and thromboembolism including ischaemic stroke. Pregnancy is a relative contraindication to thrombolysis for acute ischaemic stroke, although case reports suggest the treatment can be given in selected cases. We report a case of recurrent cryptogenic strokes in a 36-year-old female who was thrombolysed with good outcome at 37 weeks' gestation and was eventually found to have ILVNC as the cause. She had a predilection to recurrent posterior circulatory strokes due to foetal posterior communicating arteries. To our knowledge this is the first case report of safe thrombolysis for acute ischaemic stroke in pregnancy caused by ILVNC.
\end{abstract}

Keywords: left ventricular non-compaction, ischaemic stroke, stroke in pregnancy, foetal posterior communicating artery

\section{Introduction}

Stroke in the young (age $<50$ years) and stroke in pregnancy are both relatively rare but associated with significant morbidity and mortality. The underlying cause is usually not identified in up to $25 \%$ of cases of young-onset stroke and are classified as "cryptogenic stroke"; a significant proportion of cryptogenic strokes are known to be cardio-embolic in origin after more specialized investigations. ${ }^{1}$ Variants in the anatomy of the Circle of Willis, such as foetal posterior communicating arteries, can be risk factors for stroke in this population in the absence of cerebrovascular disease. $^{2}$

Isolated left ventricular non-compaction syndrome (ILVNC) is a rare form of cardiomyopathy first described in $1990,{ }^{3}$ and is characterised by prominent coarse trabeculations with deep intra-trabecular recesses in the left ventricle. These are postulated to be due to arrest of the final stages of myocardial compaction during development, giving rise to a characteristic abnormal double-layer morphology of thin compacted epicardium and a thick non-compacted endocardial layer. ILVNC is largely thought to be genetic, although recent studies showing its sudden appearance and resolution in adults suggest it could also be acquired. ${ }^{4}$ ILVNC can lead to left-ventricular thrombus formation and thus, rarely, cardio-embolic stroke, with the cumulative risk of stroke in ILVNC being approximately $15 \%$ per year. $^{5}$ 
Stroke affects approximately $0.03 \%$ of pregnancies, and can be due to ischaemia, cerebral venous sinus thrombosis, or haemorrhage, with the highest risk in the peri- and postpartum periods. ${ }^{6}$ Pregnancy is a relative exclusion criterion for treating with intravenous recombinant tissue plasminogen activator (IVTPA) in hyper-acute ischaemic stroke, and decisions to give IVTPA in pregnancy are made on a case-bycase basis. ${ }^{7}$ There is currently a paucity of evidence-based recommendations for diagnosis and secondary prevention of recurrent stroke due to ILVNC. To date there have been no case reports in the literature of safe IVTPA delivered in pregnancy for the treatment of hyper-acute ischaemic stroke due to ILVNC.

\section{Materials and Methods}

We describe a case with acute ischaemic stroke during pregnancy, on a background of recurrent strokes over the preceding two years, who was thrombolysed safely with IVTPA and was eventually found to have ILVNC as the only attributable cause for her strokes. She was also found to have foetal variants of the posterior communicating arteries.

\section{Case Presentation}

A 36-year-old female (gravida 2 para 1) has been known to our services due to bilateral cerebellar strokes 3 years previously, which were deemed cryptogenic after extensive investigations. She had been taking clopidogrel, which was switched to aspirin when she became pregnant again. Neither she nor her family had any relevant comorbid disease, and she had never taken oral contraceptives, alcohol, illicit drugs or cigarettes. She presented with recurrence of right occipital and right cerebellar strokes [superior cerebellar artery (SCA) territory] in the second trimester of the current pregnancy for which, again, no cause was found; with no firm grounds for anticoagulation, we added a prophylactic dose of lowmolecular-weight heparin (LMWH) to the aspirin.

During both these presentations, comprehensive blood panels (including thyroid function, haematinics, testing for diabetes, thrombophilia, connective tissue and auto-immune screening, serum electrophoresis, fasting homocysteine, HIV and syphilis serology, inflammatory markers, and coagulation profiles), serial Holter monitors, and transthoracic echocardiography (TTE), with one trans-oesophageal echocardiography (TOE), were repeatedly normal. Cardiac MRI was not available at that time. Contrast-enhanced angiography on magnetic resonance imaging (MRI) of the intra- and extra-cranial vessels showed stenosis of the distal basilar artery and P1 segments with bilateral foetal posterior communicating arteries but no features of vasculitis.

She presented again at 37 weeks' gestation to our emergency department with sudden-onset right-sided weakness, diplopia and dysarthria at $0800 \mathrm{~h}$ as witnessed by the husband, which occurred whilst they were en-route to work. On arrival her National Institute of Health Stroke Scale (NIHSS) score was 8 , confirmed by the attending neurologist (author DSS) at 0855h. Urgent computed tomography (CT) scan of the head was reported normal at $0935 \mathrm{~h}$. There were no other contraindications to IVTPA except for her pregnancy. After urgent multidisciplinary discussions with her obstetrician, the cardiologist (author LMN), and an independent neurologist, a clinical decision was made to administer IVTPA which was given at $1015 \mathrm{~h}$, and she was moved to the critical care unit for neuroobservation.

Her diplopia and dysarthria improved initially, but three hours later she reported right-sided paraesthesia and worsening right upper limb weakness with heaviness of the tongue, all confirmed on examination. An urgent repeat CT head scan was normal, and MRI scan of the brain revealed a $9 \mathrm{~mm}$ area of restricted diffusion in the medial aspect of the left postero-inferior cerebellum with subtle hyper-intensity on the fluid-attenuated inversion recovery (FLAIR) sequences in keeping with a hyper-acute infarct in the postero-inferior cerebellar artery (PICA) territory. She was commenced on aspirin $300 \mathrm{mg}$, and urgent bedside TTE showed no left ventricular thrombus. Repeat MRI brain scan on day 2 showed interval development of 3 new foci of restricted diffusion in different territories of the brain, so we increased LMWH to treatment dose and stopped aspirin, after which she had no further clinical events.

She underwent an uneventful caesarean section on day 4 , and on day 5 power grade had improved to $4 / 5$ in the right upper limb. However, on day 6 she redeveloped right sided facial tingling with worsening right limb weakness power $3 / 5$, and repeat MRI revealed three new small acute infarcts in the cerebellum, both PICA and SCA territories (Figure 1); we therefore added aspirin to the anticoagulation. Given the ongoing strokes despite full treatment we requested trans-oesophageal echocardiogram (TOE) which showed changes in keeping with ILVNC, further confirmed with cardiac MRI (Figure 2).

During the remainder of her admission, there were no dysrhythmias noted on critical care cardiac monitoring, 

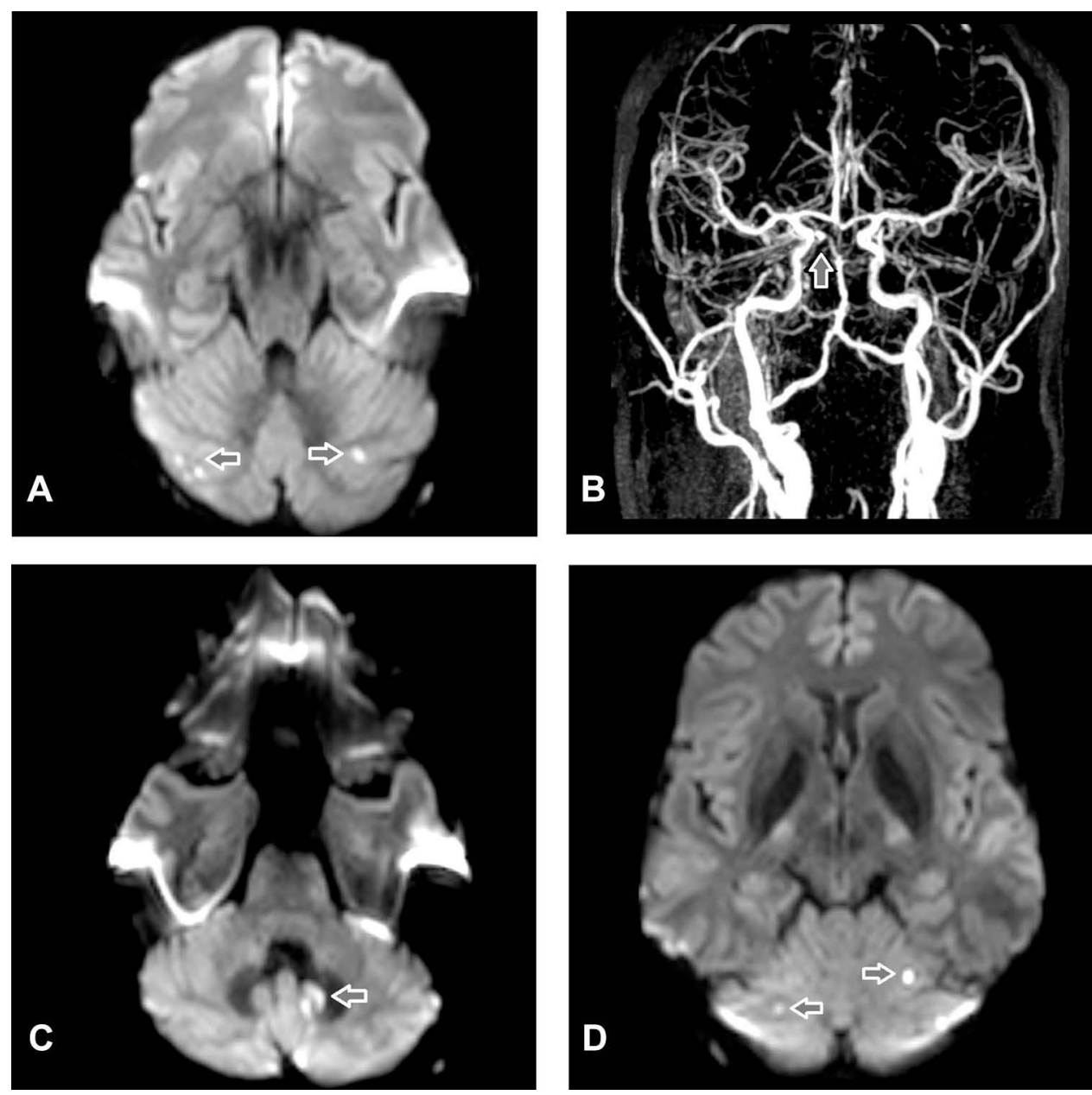

Figure I MRI brain images during acute admissions (grey arrows indicate area(s) of interest). 2015 admission: (A) diffusion weighted imaging (DWI) showing posterior circulatory artery (PCA) ischaemic strokes from 21/2 years prior; (B) contrast-enhanced MRI angiography (maximum intensity projection) showing remnant foetal PCA cerebrovasculature. 2018 admission: (C) DWI sequences of recurrence of stroke in first trimester of current pregnancy; (D) ongoing strokes during admission post-IVTPA.

and serial 24-hour Holter monitors were normal. She made a gradual recovery with appropriate neuro-rehabilitation therapy, and was discharged on day 10 with minimal right sided weakness with the ability to walk unaided. We switched her treatment to warfarin with a target international normalised ratio (INR) of 2.5-3.5, and she returned to work 2 months later with no complications since. The patient has reported compliance with the warfarin as it is more affordable in our region, with a therapeutic INR monitored by the cardiologist (author LMN). Her new infant continued to breastfeed for a total of one year and has grown normally with no sequelae noted, for which both the patient and her husband were grateful. The authors (DSS and LMN) have continued to follow up the patient to date: at almost 4 years after the IVTPA, anticoagulation initiation and ILVNC diagnosis, the child remains well and healthy as reported by the paediatricians.
Her younger brother, who had transiently immigrated to Europe during the patient's illness, was invited for review by cardiology and was also found to have asymptomatic ILVNC.

\section{Discussion}

Our case is a unique example of a combination of common stroke risk factors (pregnancy, foetal PCA remnant) with rare causes of cryptogenic stroke (ILVNC) conspiring to cause recurrent embolic strokes. The main cause of our patient's recurrent strokes over the years is chiefly attributable to the ILVNC, which is likely to be familial given the positive finding in her brother. ILVNC can be unmasked in hyperdynamic states such as pregnancy due to mechanical overloading $^{8}$ and could be why the ILVNC was not picked up on our patient previously when she was not pregnant. The imaging of choice to detect ILVNC is cardiac MRI, which we eventually pursued in our case; this modality 


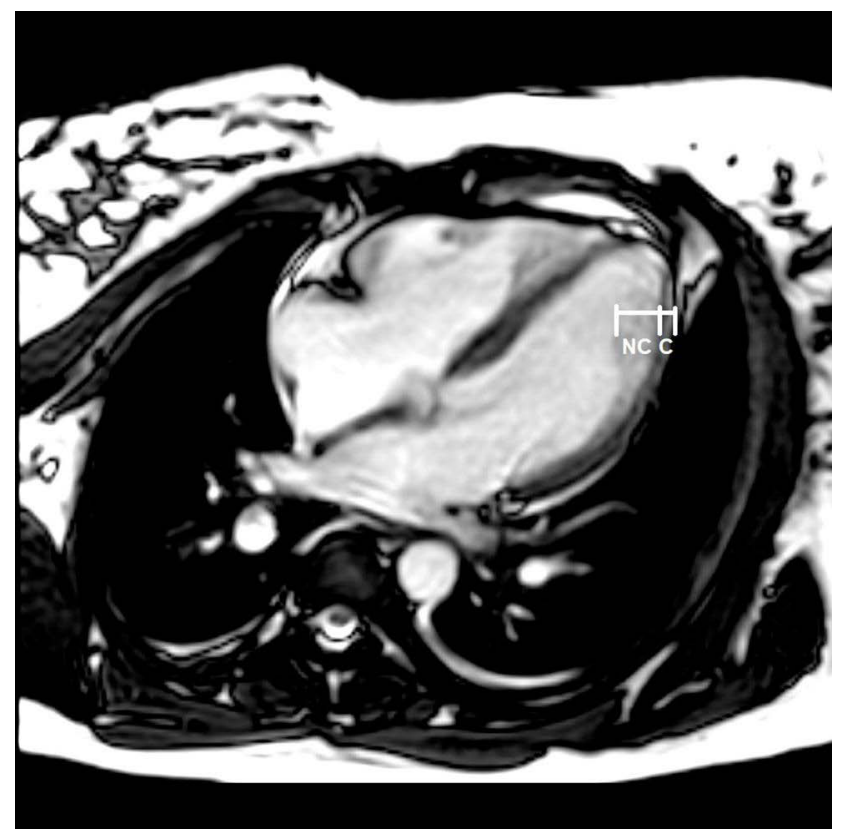

Figure 2 Cardiac MRI (pseudo-4-chamber view) revealing characteristic ILVNC morphology in segment 4-6 with non-compacted (NC)/compacted (C) ratio of $\geq 2$ : 1 .

also has the advantage of assessing myocardial fibrosis and offering prognostic information. ${ }^{9}$ One limitation to asserting ILVNC as the main contributor to the stroke presentations in our patient is that we did not have a cardiac MRI scan from her index presentation a few years prior, although it is possible that the ILVNC may not have been unmasked at that time.

Risk factors for ischaemic stroke in the context of ILVNC include an abnormal ejection fraction, visible thrombus and atrial fibrillation. ${ }^{10}$ Our patient had one of these, and additionally was pregnant which in itself is a hyper-coagulable state and may have contributed to the formation of thrombi in the trabecular recesses. Despite ongoing recurrent strokes in the first few days of this admission, TTE did not pick up on these thrombi probably due to either their small size, or from a combination of complete embolisation, spontaneous resolution, extraventricular origin of the thrombi, or dissolution after IVTPA. In our patient, there was the additional risk factor of an abnormal foetal remnant of the posterior cerebral artery, which itself is known to increase the risk of stroke ${ }^{11}$ and also explains the predilection of her cerebrovascular events for the posterior circulation.

We thrombolysed our patient after discussing risks and benefits with her, her husband, and the multi-disciplinary team including her obstetrician. The latest guidelines have recategorised pregnancy from being a contraindication to IVTPA to being a considered recommendation for the treatment when the anticipated benefits in the context of moderate or severe stroke outweigh the anticipated increased risks of uterine bleeding. ${ }^{12}$ There are case reports of successful IVTPA in ILVNC cases, ${ }^{13}$ but none during concurrent pregnancy.

Subsequent secondary prevention in ILVNC is based on expert opinion as no formal guidelines exist. Whilst antiplatelet monotherapy post-IVTPA would normally be indicated, our patient's recurrent multi-territory clinical events that were also shown on serial MRI brain scans despite standard stroke preventative therapy called for full anticoagulation, even in the absence of cardiac arrhythmias and demonstrable left ventricular thrombus. We commenced LMWH and subsequently transitioned to warfarin which is in keeping with recommendations specific to ILVNC. ${ }^{14}$

Current guidance recommends that first-degree relatives of patients with ILVNC should be screened for the condition, ${ }^{10}$ as early diagnosis can lead to treatment such as prophylactic anticoagulation to prevent thromboembolic events in high-risk patients. ${ }^{14}$ Although not relevant to our patient or her sibling, we recognise that ILVNC, as is the case with cardiomyopathies, can be associated with underlying neuromuscular disorders and patients should therefore be clinically assessed for these too when ILVNC is detected.

\section{Conclusion}

\section{What is Already Known About This Topic}

- ILVNC is a rare but important cardio-embolic cause of recurrent cryptogenic strokes.

- The diagnosis requires an index of suspicion and a comprehensive cardiological work-up, including cardiac MRI as our case illustrates.

- The pathogenic role of anomalous cerebrovasculature, such as foetal variants, in ischaemic stroke can also be unmasked during pregnancy.

\section{What This Study Adds}

- Pregnancy can exacerbate ILVNC and lead to recurrent cryptogenic strokes.

- IVTPA can be given safely in pregnancy without risk to mother or neonate in the context of embolization from ILVNC.

- Anticoagulation can be warranted but should be chosen on a case-by-case basis based on safety and efficacy profiles. 


\section{Data Sharing Statement}

The clinical history and imaging data used to support the findings of this study are included within the article.

\section{Research Ethics and Consent}

This study was conducted in accordance with the principles stated in the Declaration of Helsinki. The study is exempted from our formal institutional ethics review given it is a case report. Written informed consent for the case details to be published was obtained from the patient and has been filed in her medical notes. The authors have removed all patient identifiable information from the manuscript.

\section{Acknowledgments}

The authors would like to acknowledge the help of Dr Kevin Ombati Onyinkwa and Dr Sheila Waa, consultant radiologists at The Aga Khan University Hospital Nairobi, in obtaining the brain and cardiac MRI images.

\section{Funding}

This case series did not receive any specific funding. The cases have been compiled as part of authors' current employment under the Aga Khan University Medical College of East Africa, Faculty of Health Sciences.

\section{Disclosure}

The authors report no conflicts of interest in this work. The authors declare that there is no conflict of interest regarding the publication of this paper.

\section{References}

1. Ekker MS, Boot EM, Singhal AB, et al. Epidemiology, aetiology, and management of ischaemic stroke in young adults. Lancet Neurol. 2018;17(9):790-801. doi:10.1016/S1474-4422(18)30233-3

2. Chandra A, Li WA, Stone CR, Geng X, Ding Y. The cerebral circulation and cerebrovascular disease I: anatomy. Brain Circ. 2017;3 (2):45-56
3. Chin TK, Perloff JK, Williams RG, Jue K, Mohrmann R. Isolated noncompaction of left ventricular myocardium. A study of eight cases. Circulation. 1990;82(2):507-513. doi:10.1161/01.CIR.82.2. 507

4. Okumura TMT. Unsolved issue in left ventricular noncompaction: is the strange form of myocardium congenital or acquired? Cardiology. 2019;26:1-2

5. Stollberger C, Blazek G, Dobias C, Hanafin A, Wegner C, Finsterer J. Frequency of stroke and embolism in left ventricular hypertrabeculation/noncompaction. Am $\quad J$ Cardiol. 2011;108 (7):1021-1023. doi:10.1016/j.amjcard.2011.05.039

6. Swartz RH, Cayley ML, Foley N, et al. The incidence of pregnancy-related stroke: a systematic review and meta-analysis. Int J Stroke. 2017;12(7):687-697. doi:10.1177/1747493017723271

7. Watanabe TT, Ichijo M, Kamata T. Uneventful pregnancy and delivery after thrombolysis plus thrombectomy for acute ischemic stroke: Case Study and literature review. J Stroke Cerebrovasc 315 Dis. 2019;28(1):70-75. doi:10.1016/j.jstrokecerebrovasdis.2018.09.002

8. Gati S, Papadakis M, Papamichael ND, et al. Reversible de novo left ventricular trabeculations in pregnant women: implications for the diagnosis of left ventricular noncompaction in low-risk populations. Circulation. 2014;130(6):475-483. doi:10.1161/ CIRCULATIONAHA.114.008554

9. Choi Y, Kim SM, Lee SC, Chang SA, Jang SY, Choe YH. Quantification of left ventricular trabeculae using cardiovascular magnetic resonance for the diagnosis of left ventricular non-compaction: evaluation of trabecular volume and refined semi-quantitative criteria. J Cardiovasc Magn Reson. 2016;18 (1):24. doi:10.1186/s12968-016-0245-2

10. Towbin JA, Lorts A, Jefferies JL. Left ventricular non-compaction cardiomyopathy. Lancet. 2015;386(9995):813-825. doi:10.1016/ S0140-6736(14)61282-4

11. Lambert SL, Williams FJ, Oganisyan ZZ, Branch LA, Mader EC Jr. Fetal-type variants of the posterior cerebral artery and concurrent infarction in the major arterial territories of the cerebral hemisphere. J Investig Med High Impact Case Rep. 2016;4 (3):2324709616665409.

12. Powers WJ, Rabinstein AA, Ackerson T, et al. Guidelines for the early management of patients with acute ischemic stroke: 2019 update to the 2018 guidelines for the early management of acute ischemic stroke: a guideline for healthcare professionals from the American Heart Association/American Stroke Association. Stroke. 2019;50(12):e344-e418.

13. Finsterer J, Stöllberger C, Sodeck G. Thrombolysis of ischemic stroke from noncompaction in metabolic myopathy. Neurologist. 2012;18(5):296-297. doi:10.1097/NRL.0b013e318266f5db

14. Di Fusco SA, Luca F, Madeo A, et al.Left ventricular noncompaction: diagnostic approach, prognostic evaluation, and management strategies. Cardiol Rev. 2020;28(3):125-134. doi:10.1097/CRD.0000000000000251

\section{Publish your work in this journal}

The International Medical Case Reports Journal is an international, peer-reviewed open-access journal publishing original case reports from all medical specialties. Previously unpublished medical posters are also accepted relating to any area of clinical or preclinical science. Submissions should not normally exceed 2,000 words or 4 published pages including figures, diagrams and references. The manuscript management system is completely online and includes a very quick and fair peer-review system, which is all easy to use. Visit http://www.dovepress.com/testimonials.php to read real quotes from published authors. 